\title{
Genotypic Assessment of Bacterial Leaf Blight Resistance in Indigenous Rice (Oryza sativa L.) Germplasm
}

\author{
R. Ashiba ${ }^{1}$, K. Eraivan Arutkani Aiyanathan ${ }^{1}$, R. Kannan $^{1}$ and M. Arumugam Pillai ${ }^{2}$ \\ ${ }^{1}$ Department of Plant Pathology, ${ }^{2}$ Department of Plant Breeding and Genetics, \\ Agricultural College \& Research Institute, Tamilnadu Agricultural University, \\ Killikulam - 628252, Tamilnadu, India \\ *Corresponding author
}

\section{A B S T R A C T}

\begin{tabular}{|l|}
\hline Ke y w or d s \\
Rice germplasm, \\
BLB, Resistance, \\
Genetic diversity, \\
Cluster analysis \\
\hline Article Info \\
\hline $\begin{array}{l}\text { Accepted: } \\
\text { 05 June } 2020 \\
\text { Available Online: } \\
10 \text { July } 2020\end{array}$ \\
\hline
\end{tabular}

One hundred rice genotypes were analyzed to evaluate the genetic polymorphism and identification of resistant lines to bacterial leaf blight (BLB) disease caused by Xanthomonas oryzae pv. oryzae using simple sequences repeat (SSR) markers. A total of 38 alleles were detected by seven polymorphic markers showing highly polymorphic across all genotypes with an average of 5.42 alleles per polymorphic marker. The marker RM-21, RM-122 and RM-224 produced maximum 6 alleles. The PIC values ranged from 0.345 to 0.688 and marker RM-21 was found to be the most appropriate marker to discriminate BLB resistant rice genotypes owing to the highest PIC value of 0.688 . The cluster analysis showed that these genotypes grouped into four clusters, in which cluster IV had maximum forty genotypes followed by cluster III and cluster I. Multiple resistance (B) genes $(X a 21+x a 13+x a 5+X a 4)$ were identified in germplasms such as Dhalakeera, Swarnamasuri, Purple puttu, Veethiruppu showing high level of resistance to BLB, while Navarai black 5571 and Kalyani were found to be moderately resistant to BLB disease in both field and controlled condition. These genetically diverse BLB resistant genotypes can be directly utilized in rice BLB resistance breeding programs.

\section{Introduction}

The world's most daring problem is to nourish the developing population which is expected to reach 8 billion individuals by 2020, due to expanding population (Kubo and Purevdorj, 2004). As the population builds, the production of food diminishes due to an absence of reasonable land for crop development. The infection caused due to bacterial pathogens becomes a major challenge for the rice breeders. BLB is a vascular disease that causes a white yellow discoloration in rice crop along veins, leaf margins, leaf blades and those lesions may extend to the sheath (Gnanamanickam et al., 1999).

In rice it causes annual yield losses conservatively estimated at 50\% (Song and Goodman, 2001). When the rice is infected by Xanthomonas oryzae pv. oryzae, although the 
symptoms of ailment may be determined at tillering stage, the disease can also retain to increase as the plant grows. It is observed that the rice plant at much less than 21 days old are more liable to disease and the bacteria may additionally desire temperature at 28$34^{\circ} \mathrm{C}$ for boom.

The improvement of host resistance and utility of chemical and organic measures have been used for the management of BLB (Akhtar et al., 2008). However, for the BLB management, host plant resistance is the most appropriate one to manage the pathogen.

To date, a total of 42 BLB resistance genes ( $R$ genes) have been identified in rice including $X a 1, X a 2, X a 3 / X a 26, X a 4, X a 5, X a 6, X a 7$, $x a 8, x a 9, X a 10, X a 11, X a 12, x a 13, X a 14$, xa15, Xa16, Xa17, Xa18, xa19, xa20, Хa21, $X a 22(\mathrm{t}), \quad X a 23, \quad x a 24(\mathrm{t}), \quad X a 25, \quad x a 26(\mathrm{t})$, $X a 27, x a 28(\mathrm{t}), \quad X a 29(\mathrm{t}), \quad X a 29(\mathrm{t}), \quad X a 31(\mathrm{t})$, $X a 33(\mathrm{t}), \quad x a 34(t), X a 35(t), X a 36(t), X a 37$, $X a 38, X a 39, X a 40, x a 41(t), X a 42$. The genes of recessive resistance comprise $x a 5, x a 8$, $x a 9, \quad x a 13, \quad x a 15, x a 19, \quad x a 20, x a 24$, $x a 25 / X a 25(t), x a 26(\mathrm{t}), x a 28(\mathrm{t}), x a 31(\mathrm{t})$, and $x a 33(\mathrm{t})$.Ten of the recessive $\mathrm{R}$ genes; $x a 5$ (Petpisit et al., 1977), xa8(Singh et al., 2002), xa13 (Ogawa et al., 1987), xa24 (Khush and Angeles, 1999), xa26, xa28(Lee et al., 2003)and xa32 (Ruan et al., 2008) confer race-specific resistance.

While the other three genes viz.,xa15(Ogawa 1996), xa19 and xa20 (Taura et al., 1992), were created by mutagenesis and each confers a wide spectrum of resistance to Xoo (Ogawa, 1996; Lee et al., 2003; Chen et al., 2002).

Determination of genetic diversity can be done by assessing morphological or molecular data. The use of advanced molecular technologies is one of the possible approach to understand their diversity. Evaluation of genetic diversity using DNA marker technology is non-destructive, not affected by environmental factors, requires small number of samples, and does not require large experimental setup and equipment's for measuring physiological parameters (Kanawapee et al., 2011).

Simple sequence repeat (SSR) marker analysis is an important tool for the identification of genetic variation in accessions(Sajib et al., 2012; Ma et al., 2011). SSR markers are highly informative, mostly monolocus, codominant, easily analyzed and cost effective (Garcia et al., 2004) and able to detect high level of allelic diversity (Ni et al., 2002), thus being widely applied in genetic diversity analysis, molecular map construction and gene mapping(Zhang et al., 2007; Ma et al., 2011) and analysis of germplasm diversity (Zhou et al., 2003; Jin et al., 2010); Ma et al., 2011).

SSR markers even in less number can give a better genetic diversity spectrum due to their multi-allelic and highly polymorphic nature(Singh et al., 2016). Therefore the present study was undertaken with the aim to identify BLB resistant rice lines and molecular diversity in rice genotypes using SSR markers.

\section{Materials and Methods}

\section{Plant materials}

Seeds of one hundred number of rice genotypes (Table 1) were obtained from Department of Plant breeding and Genetics, Agricultural College and Research Institute (AC\&RI), Killikulam, Thoothukudi District, together with the comparison line IRBB60 (carrying Xa21, xa13, xa5, Xa4) as positive resistant gene check and $\mathrm{TN} 1$ as negative gene check for the study. All these lines were grown in field as well as in pots in the glasshouse. 


\section{Bacterial isolate and culture media}

The virulent isolate of Xanthomonas oryzae pv. oryzae (Xoo) was collected from Tamil Nadu Agricultural University, Coimbatore. The Xoo isolate was multiplied and maintained on Xoo specific modified Wakimoto's medium, which contains sucrose $20 \mathrm{~g}$, sodium phosphate $\quad 0.82 \mathrm{~g}$, ferrous sulphate $0.05 \mathrm{~g}$, calcium nitrate $0.5 \mathrm{~g}$, peptone 5.0g, agar $17.0 \mathrm{~g}$ and distilled water $\quad 1000$ $\mathrm{ml}$. The prepared medium was transferred to triangular flasks. The flasks were closed with cotton plugs, covered with kraft paper, and autoclaved at $121^{\circ} \mathrm{C}$ for 30 minutes.

\section{Inoculum preparation and plant inoculation}

For inoculum preparation, $10 \mathrm{ml}$ of sterile distilled water poured into pathogenic bacterial culture and preserved the inoculum concentration at @10 $\mathrm{CFU} / \mathrm{ml}$. Before panicle initiation, plants with entirely fresh and expanded leaves were inoculated using the leaf cutting process(Kauffman, 1973). The sterile scissors were dipped into the inoculum and 3 leaves per plant were trimmed from the tip of growing leaf about $2-3 \mathrm{~cm}$ apart. BLB lesions were found 15 days after inoculation on the clipped leaves.

\section{Evaluation}

During the Rabi season of 2019 -2020, the screening was performed in the rice crop at 10, 30, 50 days after planting under field and controlled conditions. The Percent Disease Index (PDI) and scales for evaluating the BLB resistance under field condition was determined based on the method suggested by Nagendran et al., (2013)

Percent Disease Index $(\mathrm{PDI})=$

$\frac{\text { Sum of all Numerical Ratings }}{\text { Total No of leaves graded }} \times \frac{100}{\times \text { Maximum Grade obtained }}$

The scoring system used to evaluate breeding lines for BLB resistance in the field (IRRI, 1996; Rafi et al., 2013) as:

\begin{tabular}{|l|c|l|}
\hline Scale & $\begin{array}{c}\text { Disease Leaf } \\
\text { Area }(\mathbf{\%})\end{array}$ & Description \\
\hline $\mathbf{0}$ & 0 & Immune \\
\hline $\mathbf{1}$ & $1-10$ & Resistant \\
\hline $\mathbf{3}$ & $11-25$ & $\begin{array}{l}\text { Moderate } \\
\text { resistant }\end{array}$ \\
\hline $\mathbf{5}$ & $26-50$ & $\begin{array}{l}\text { Moderate } \\
\text { susceptible }\end{array}$ \\
\hline $\mathbf{7}$ & $51-75$ & Susceptible \\
\hline $\mathbf{9}$ & $76-100$ & $\begin{array}{l}\text { Highly } \\
\text { susceptible }\end{array}$ \\
\hline
\end{tabular}

The scoring system used to evaluate breeding lines for BLB resistance in the glasshouse (IRRI, 1996) as:

\begin{tabular}{|c|c|c|}
\hline SI.No & $\begin{array}{l}\text { Lesion length } \\
\text { (cm) }\end{array}$ & Description \\
\hline 1. & $0-5$ & Resistant \\
\hline 2. & $5-10$ & $\begin{array}{l}\text { Moderately } \\
\text { resistant }\end{array}$ \\
\hline 3. & $10-15$ & $\begin{array}{l}\text { Moderately } \\
\text { susceptible }\end{array}$ \\
\hline 4. & $>15$ & Susceptible \\
\hline
\end{tabular}

\section{Genomic DNA extraction}

DNA samples were extracted from young leaves (21 days old seedlings) using the Cetyl-trimethyl ammonium bromide (CTAB) method modified from the protocol of Doyle and Doyle (1990). The quality of genomic DNA was analyzed in $0.8 \%$ agarose gel. Total genomic DNA samples were diluted to $100 \mathrm{ng} / \mu \mathrm{l}$ using Tris HCL buffer and stored at $-20^{\circ} \mathrm{C}$ till further use.

\section{SSR markers and PCR amplification}

Of the 10 SSR markers screened, 7 markers showed polymorphism (Table 2). 
Amplification of DNA fragments was carried out using gene specific markers of Xa21, xa13, xa5, Xa4. Each PCR amplification reaction was in a total volume of $10 \mu \mathrm{l}$ containing $5 \mu \mathrm{l}$ of $2 \mathrm{X} \mathrm{Taq}$ polymerase master mix, $0.5 \mu 1$ of DNA, $0.3 \mu 1$ of forward primer, $0.3 \mu l$ of reverse primer, $3.9 \mu l$ of water. The polymerase chain reaction was performed in a thermocycler with the following cycles: the initial denaturation at $94^{\circ} \mathrm{C}$ for 5 min followed by 35 cycles of denaturation at $94^{\circ} \mathrm{C}$ for 30 sec, annealing at $55^{\circ} \mathrm{C}$ for $30 \mathrm{sec}$ followed by extension at $72^{\circ} \mathrm{C}$ for $1 \mathrm{~min}$ and final extension at $72^{\circ} \mathrm{C}$ for $10 \mathrm{~min}$. In order to determine polymorphism, PCR products were checked on $3 \%$ agarose in $1 \mathrm{X}$ TBE buffer. For pre-staining, ethidium bromide was added to the gel at the concentration of $10 \mu 1 / \mathrm{ml}$ before the gel was poured. The samples were run on the gel at $140 \mathrm{~V}$ until the bromophenol blue dye migrated almost to the end of the gel. Thereafter electrophoresis, gel documentation was carried out and identified for the presence (++) and absence (--) of BLB gene linked DNA fragment.

\section{Data analysis}

The amplified fragments of all the rice genotypes were scored by comparing with respective resistance (IRBB60) and susceptible (TN1) bands. The data was scored using "++/--" signs for the presence/absence of target gene, respectively.

\section{Cluster analysis based on UPGMA}

The binary data matrix generated by polymorphic SSR markers was subjected to further analysis using Darwin software version 6.0. The dissimilarity matrix was used as an input for analysis of clusters. Phylogenetic tree was formed following unweighted pair group method of arithmetic means (UPGMA) using the tool. In unweighted pair-group average (UPGMA) clusters are joined based on the average distance between all members in four groups.

\section{Polymorphic information content (PIC)}

PIC for SSR markers was calculated as per the formula:

$$
\mathrm{PIC}=1-\sum P_{i j}^{2}
$$

Where, PIC $i j$ is the frequency of the $j^{\text {th }}$ allele for the $i^{\text {th }}$ marker and summation extends over $\mathrm{k}$ alleles.

\section{Results and Discussion}

Screening for BLB resistance of rice germplasm under field and controlled conditions

Bacterial leaf blight of rice has been reported in several parts of the world with high incidence and severity (Sonti, 1998). Therefore strategies adapted to particular environments must be developed to avoid possible epidemics. Knowledge of varietal resistance is important for selecting cultivars with durable resistance to the disease (Banito et al., 2010). The results of the present study showed the various levels of resistance to bacterial leaf blight in rice germplasm.

One hundred number of rice germplasm were screened for BLB resistance under field conditions without any artificial inoculation. The percentage of germplasm found to be resistant, moderately resistant, moderately susceptible and susceptible on 50 days after planting under field screening was found to be three per cent, thirty nine per cent, forty seven per cent and twelve per cent, respectively. Among the resistant genotypes Dhalakeera, Purple puttu and Veethiruppu recorded lowest PDI value of 8.90, 9.10 and 9.50 respectively. In this study, three resistant varieties i.e. Purpleputtu, Dhalakeera and Veethiruppu 
were found to be completely resistant in field conditions. They are recommended for planting in areas where BLB often occurs to cause reduction in yield. However, the responses of these varieties should be further confirmed by artificial screening.

The rice germplasm was evaluated for its BLB resistance under controlled glass house environment using clipping method for the uniform spread of pathogen in plant tissues. The percentage of germplasm found to be resistant, moderately resistant, moderately susceptible and susceptible under artificial screening was two per cent, thirty eight per cent, forty three per cent and sixteen per cent, respectively. Among the resistant genotypes, Swarnamasuri and Purple puttu recorded mean lesion length of 4.38 and $4.96 \mathrm{~cm}$, respectively. As expected, the resistance level among germplasmwas low with more than half of the resources being moderately susceptible or susceptible (Table 3).

\section{Molecular screening for BLB resistance genes using SSR markers}

The indigenous lines of 100 rice genotypes were evaluated for the presence and absence of BLB resistance genes viz., Xa21, xa13, $x a 5, X a 4$ using PCR based gene linked markers (Table 4). Molecular base pairs for total seven SSR markers which corresponds to IRBB60 (positive control) and TN1 (negative control) was given in the Table 2. During the gene survey using RM21 marker, out of 100 genotypes, 30 genotypes along with a positive control amplified 190bp size fragments which indicated the presence of $X a 21$ gene. While the remaining 70 genotypes amplified 180 bp DNA fragments which showed the absence of $\mathrm{Xa21}$ gene. DNA analysis of $x a 13$ resistance gene in all the selected rice germplasm exhibited the presence of bands with markers RM230 and RM264. With marker RM230, 38 genotypes along with a positive control amplified 280bp size fragments, while with marker RM264, 35 genotypes along with a positive control amplified $180 \mathrm{bp}$ indicating the presence of $x a 13$ gene. DNA analysis of $x a 5$ resistance gene in all the selected rice germplasm exhibited the presence of bands with SSR markers RM122 and RM164. Out of 100 genotypes, 24 genotypes amplified 240bp corresponding to positive control (IRBB60) which showed the presence of $x a 5$ specific bands with marker RM122.

While with the marker RM164, 21 genotypes along with positive control amplified $240 \mathrm{bp}$ indicating the presence of $x a 5$ gene. Ramalingam et al., (2001), Lee et al., (2003)and Kihupi et al., (2001)also conducted similar type of polymorphic survey for the presence of $x a 5, x a 13$ and $\mathrm{Xa21}$ genes in rice germplasm. DNA analysis of the genotypes with RM224 and RM167 markers exhibited the presence and absence of $\mathrm{Xa} 4$ gene. With marker RM 224, 46 genotypes showed the amplicon of $160 \mathrm{bp}$ corresponding to resistant allele and hence considered as resistant genotypes. While with marker RM 167, out of 100 rice genotypes, 11 genotypes along with positive control amplified $140 \mathrm{bp}$ size fragments which indicated the presence of $\mathrm{Xa} 4$ gene. Similar type of polymorphic survey was done by Arif et al., (2008)for the presence (++) and absence (--) of Xa4 gene in rice germplasmin Pakistan.

The resistant genotype Dhalakeera exhibited the presence of positive bands with the markers RM21, RM230, RM224 and RM167. Swarnamasuri exhibited the presence of positive bands for the markers RM21, RM230, RM264 and RM164. Further, Purple puttu and Veethiruppu exhibited the presence of positive bands with the markers RM21, RM264, RM164 and RM224. In the present study, four BLB resistant genes were identified, in which 7 genotypes carrying the 
combination of $X a 21+x a 13+x a 5$ genes, while 10genotypes carrying the combination of $X a 21+x a 13+X a 4$ genes. Further, 7 genotypes were identified with combination of $X a 21+x a 5+X a 4$ genes and 12 genotypes carrying the combination of $x a 13+x a 5+X a 4$ genes.The lines carrying four $\mathrm{R}$ genes i.e., $(X a 21+x a 13+x a 5+X a 4)$ are Kalyani, Navarai black 5571, Purple puttu, Veethiruppu, along with resistant check IRBB60(Table 5). It is worthy of mention that the resistance levels of Dhalakeera, Swarnamasuri and Purple puttu, Veethiruppu were found to be high due to the presence of multi genes $(X a 21+x a 13+X a 4)$, $(X a 21+x a 13+x a 5)$ and $(X a 21+x a 13+$ $x a 5+X a 4)$, respectively. Navarai black 5571 and kalyani carrying four $\mathrm{R}$ genes showed moderate resistance to BLB under field and artificial screening.

The rice genotypes carrying multi-genes will pave way for the identification of resistant sources for further breeding programmes. Similar results were reported by Sodhi et al., (2003)concluding that combination of $\mathrm{Xa21}$ with $x a 13$ and $X a 5$ BLB resistance genes are effective against the prevalent strains of Xanthomonas (Xoo). Perumalsamy et al., (2010)reported that the genes Xa4, xa5 along with $X a 21$ provide wide spectrum of bacterial leaf blight resistance against many $X$. oryzaeraces.

\section{Polymorphism and marker efficiency}

All the 100 germplasm lines were genotyped by using 7 SSR markers. The allele size variation between the smallest and the largest allele at a given SSR was correlated with number of alleles per locus (Table 6). Thus, RM 167 presented the smallest allele size range $100-150 \mathrm{bp}$ and $\mathrm{RM} 122$ had the largest allele size range $240-300 \mathrm{bp}$. The informativeness of the markers was revealed by calculating the polymorphism information content (PIC). The PIC value of seven SSR markers ranged from 0.345 (RM 167) to 0.688 (RM 21) with 0.528 average respectively. Above 0.5 values were observed for RM 21 (0.688), RM 230 (0.507), RM 64 (0.664), RM 122 (0.501) and RM 224 (0.603) which showed the high polymorphic nature of primers. Similar studies proved the high informativeness of SSR markers (Kumar and Bhagwat, (2012) and Lang et al., (2014).

\section{Cluster analysis}

A phylogenetic tree (Fig. 1) based on neighbor joining method was constructed using UPGMA. The rice genotypes were grouped into four main clusters (Table 7) i.e. cluster I, cluster II, cluster III and cluster IV consisting 25, 10, 25 and 40 genotypes, respectively. Cluster analysis was done to identify the variation between the genotypes and within the genotypes. The resistant genotypes are present in different cluster group having high dissimilarity than other genotypes. It clearly notifies that resistant genotypes had high diversity with BLB resistance.

Genetic diversity is the key determinant of germplasm utilization in crop improvement. Population with high level of genetic variation is the valuable resource for broadening the genetic base in any breeding program. The study of genetic variability was estimated based on the amplification pattern of 7 SSR markers. Brondani et al., (2006) reported the relative efficiency of utilizing the SSR markers for the assessment of genetic diversity. In this study genetic diversity among the accessions were evaluated by model based on clustering approach using the SSR genotypic data. The clusters were developed by using neighbor joining method. This grouping method was further supported by studies of Upadhyay et al., (2012), Nachimuthu et al., (2015) and Singh et al., (2016). 
Table.1 List of rice genotypes included in current study

\begin{tabular}{|c|c|c|c|c|c|c|c|}
\hline S.No. & Germplasms & S.No. & Germplasms & S.No. & Germplasms & S.No. & Germplasms \\
\hline 1 & $\begin{array}{l}\text { TN1(Susceptible } \\
\text { check) }\end{array}$ & 26 & Chittini 5520 & 51 & Maranellu & 76 & Swarnamasuri \\
\hline 2 & $\begin{array}{l}\text { IRBB60 } \\
\text { (Resistant } \\
\text { check) }\end{array}$ & 27 & $\begin{array}{l}\text { Chuvannachittini } \\
7135\end{array}$ & 52 & Mallikar & 77 & Thondi \\
\hline 3 & Adukan & 28 & Chomala 826 & 53 & Mattai & 78 & Thuyamalli \\
\hline 4 & Aman & 29 & $\begin{array}{l}\text { Company } \\
\text { thavalaikannan }\end{array}$ & 54 & Molikarumbu & 79 & Uma \\
\hline 5 & Anjali & 30 & Dhalakeera & 55 & Mapillai samba & 80 & Varakuranellu \\
\hline 6 & Annada & 31 & Jai sree ram & 56 & Mulampunchan & 81 & Vanaprabha \\
\hline 7 & Aryan 917 & 32 & $\begin{array}{l}\text { Kunjukunju } \\
7168\end{array}$ & 57 & Navarai & 82 & Vattan 5052 \\
\hline 8 & Aryan 1023 & 33 & $\begin{array}{l}\text { Kunjukunju } \\
6974\end{array}$ & 58 & Navarai Black & 83 & Veethiruppu \\
\hline 9 & Aryan 1102 & 34 & $\begin{array}{l}\text { Kunjukunju } \\
1811\end{array}$ & 59 & $\begin{array}{l}\text { Navarai Black } \\
5571\end{array}$ & 84 & Virendra \\
\hline 10 & Aryan 1203 & 35 & Karnellu & 60 & $\begin{array}{l}\text { Navarai Black } \\
957\end{array}$ & 85 & White ponni \\
\hline 11 & Aryan 5532 & 36 & Karukot & 61 & $\begin{array}{l}\text { Navarai Black } \\
6263\end{array}$ & 86 & Thamarai \\
\hline 12 & Aryan 6333 & 37 & Karuvalli & 62 & Noothipattu & 87 & Salem sannam \\
\hline 13 & Bharathi & 38 & KaruthaNavara & 63 & Norugan & 88 & $\begin{array}{l}\text { Vasanai } \\
\text { samba }\end{array}$ \\
\hline 14 & Bommi & 39 & Kalyani & 64 & Ohenellu 6305 & 89 & Anna 4 \\
\hline 15 & Chemban 986 & 40 & Kullakar & 65 & Oheruchittini & 90 & Chenellu 6805 \\
\hline 16 & Chandikar & 41 & Kallondaikar & 66 & Pattani & 91 & Chungamnellu \\
\hline 17 & Chembaru 5599 & 42 & Kattanoor & 67 & Purple puttu & 92 & Kodikannan \\
\hline 18 & Chembaru 4331 & 43 & Kalinga & 68 & Poongar & 93 & Varapudha \\
\hline 19 & ChittiraiKar & 44 & Koltara samba & 69 & Rajalakshmi & 94 & $\begin{array}{l}\text { Krishna } \\
\text { hemarathi }\end{array}$ \\
\hline 20 & $\begin{array}{l}\text { Chiruchittini } \\
882\end{array}$ & 45 & $\begin{array}{l}\text { Kothamalli } \\
\text { samba }\end{array}$ & 70 & Sadabahar & 95 & Jaya \\
\hline 21 & Chenellu 4735 & 46 & Kichali samba & 71 & Shabhagidan & 96 & Abiyan \\
\hline 22 & Chenellu 5590 & 47 & Kayamma & 72 & Seeraga samba & 97 & Namchenbyeo \\
\hline 23 & Chenthodi & 48 & Kuliyadichan & 73 & Surakuruvai & 98 & Gowni \\
\hline 24 & $\begin{array}{l}\text { Chenkayamma } \\
5523\end{array}$ & 49 & Kerala kandasala & 74 & Swarna & 99 & Karsamba \\
\hline 25 & Chittini 1123 & 50 & Kalakeerai & 75 & Swarnamalli & 100 & White sannam \\
\hline
\end{tabular}


Table.2 List of gene-specific SSR markers used for screening of BLB resistance genes

\begin{tabular}{|c|c|c|c|c|c|c|}
\hline Gene & $\begin{array}{l}\text { Primer } \\
\text { name }\end{array}$ & Primer Sequence (5'-3') & $\begin{array}{l}\text { No of } \\
\text { Base } \\
\text { pairs }\end{array}$ & $\begin{array}{l}\text { Annealing } \\
\text { temp }\left({ }^{\circ} \mathrm{C}\right)\end{array}$ & $\begin{array}{l}\text { Resistant } \\
\text { band (bp) }\end{array}$ & $\begin{array}{l}\text { Susceptible } \\
\text { band (bp) }\end{array}$ \\
\hline \multirow[t]{2}{*}{$X a 21$} & \multirow[t]{2}{*}{ RM21 } & F: ACAGTATTCCGTAGGCACGG & 20 & & & \\
\hline & & R: GCTCCATGAGGGTGGTAGAG & 20 & 50 & 190 & 180 \\
\hline \multirow[t]{4}{*}{$x a 13$} & \multirow[t]{2}{*}{ RM230 } & F: GCCAGACCGTGGATGTTC & 18 & & & \\
\hline & & R: CACCGCAGTCACTTTTCAAG & 20 & 50 & 280 & 270 \\
\hline & \multirow[t]{2}{*}{ RM264 } & F: GTTGCGTCCTACTGCTACTTC & 21 & & & \\
\hline & & R: GATCCGTGTCGATGATTAGC & 20 & 50 & 180 & 190 \\
\hline \multirow[t]{4}{*}{$x a 5$} & \multirow[t]{2}{*}{ RM122 } & F: GAGTCGATGTAATGTCATCAGTGC & 24 & & & \\
\hline & & R: GAAGGAGGTATCGCTTTGTTGGAC & 24 & 55 & 240 & 270 \\
\hline & \multirow[t]{2}{*}{ RM164 } & F: TCTTGCCCGTCACTGCAGATATCC & 24 & & & \\
\hline & & R: GCAGCCCTAATGCTACAATTCTTC & 24 & 55 & 240 & 250 \\
\hline \multirow[t]{4}{*}{ Xa4 } & \multirow[t]{2}{*}{ RM224 } & F: ATCGATCGATCTTCACGAGG & 20 & & & \\
\hline & & R: TGCTATAAAAGGCATTCGGG & 20 & 55 & 160 & 150 \\
\hline & \multirow[t]{2}{*}{ RM167 } & F: GATCCAGCGTGAGGAACACGT & 21 & & & \\
\hline & & R: AGTCCGACCACAAGGTGCGTTGTC & 24 & 55 & 140 & 130 \\
\hline
\end{tabular}

Table.3 Screening of rice germplasm under field and controlled conditions

\begin{tabular}{|c|c|c|c|c|c|c|}
\hline \multirow[t]{2}{*}{ S.no } & \multirow[t]{2}{*}{ Germplasms } & \multicolumn{3}{|c|}{ Field screening } & \multicolumn{2}{|c|}{ Artificial screening } \\
\hline & & PDI & Scale & Description & $\mathbf{L L}(\mathbf{c m})$ & Description \\
\hline 1. & TN 1 & 45.70 & 5 & MS & 18.75 & $\mathrm{~S}$ \\
\hline 2. & IRBB60 & 12.30 & 3 & MR & 6.25 & MR \\
\hline 3. & Adukan & 20.80 & 3 & MR & 10.00 & MR \\
\hline 4. & Aman & 30.20 & 5 & MS & 14.50 & MS \\
\hline 5. & Anjali & 30.30 & 5 & MS & 9.50 & MR \\
\hline 6. & Annada & 30.60 & 5 & MS & 7.50 & MR \\
\hline 7. & Aryan 917 & 54.10 & 7 & $\mathrm{~S}$ & 16.25 & $\mathrm{~S}$ \\
\hline 8. & Aryan 1023 & 55.00 & 7 & $\mathrm{~S}$ & 11.25 & MS \\
\hline 9. & Aryan 1102 & 63.50 & 5 & MS & 9.50 & MR \\
\hline 10. & Aryan 1203 & 23.70 & 3 & MR & 7.50 & MR \\
\hline 11. & Aryan 5532 & 24.40 & 3 & MR & 8.25 & MR \\
\hline 12. & Aryan 6333 & 30.40 & 5 & MS & 13.50 & MS \\
\hline 13. & Bharathi & 50.00 & 5 & MS & 14.50 & MS \\
\hline 14. & Bommi & 30.80 & 5 & MS & 11.75 & MS \\
\hline 15. & Chemban 986 & 15.20 & 3 & MR & 7.25 & MR \\
\hline 16. & Chandikar & 25.60 & 3 & MR & 11.25 & MS \\
\hline 17. & Chembaru 5599 & 30.90 & 5 & MS & 9.50 & MR \\
\hline 18. & Chembaru 4331 & 69.70 & 7 & $\mathrm{~S}$ & 17.25 & $\mathrm{~S}$ \\
\hline 19. & ChittiraiKar & 43.20 & 5 & MS & 10.00 & MR \\
\hline 20. & Chiruchittini 882 & 40.10 & 5 & MS & 8.25 & MR \\
\hline 21. & Chenellu 4735 & 21.20 & 3 & MR & 14.75 & MS \\
\hline
\end{tabular}




\begin{tabular}{|c|c|c|c|c|c|c|}
\hline 22. & Chenellu 5590 & 20.50 & 3 & MR & 6.25 & MR \\
\hline 23. & Chenthodi & 60.60 & 7 & $\mathrm{~S}$ & 14.25 & MS \\
\hline 24. & Chenkayamma 5523 & 17.80 & 3 & MR & 12.14 & MS \\
\hline 25. & Chittini 1123 & 40.70 & 5 & MS & 16.25 & $\mathrm{~S}$ \\
\hline 26. & Chittini 5520 & 20.50 & 3 & MR & 12.50 & MS \\
\hline 27. & $\begin{array}{l}\text { Chuvannachittini } \\
7135\end{array}$ & 47.50 & 5 & MS & 14.25 & MS \\
\hline 28. & Chomala 826 & 35.60 & 5 & MS & 14.60 & MS \\
\hline 29. & $\begin{array}{l}\text { Company } \\
\text { thavalaikannan }\end{array}$ & 65.80 & 7 & $\mathrm{~S}$ & 18.25 & $\mathrm{~S}$ \\
\hline 30. & Dhalakeera & 8.90 & 1 & $\mathrm{R}$ & 5.47 & MR \\
\hline 31. & Jai sree ram & 71.80 & 7 & $\mathrm{~S}$ & 9.50 & MR \\
\hline 32. & Kunjukunju 7168 & 48.10 & 5 & MS & 13.50 & MS \\
\hline 33. & Kunjukunju 6974 & 30.50 & 5 & MS & 14.75 & MS \\
\hline 34. & Kunjukunju 1811 & 20.80 & 3 & MR & 9.75 & MR \\
\hline 35. & Karnellu & 40.90 & 5 & MS & 17.75 & $\mathrm{~S}$ \\
\hline 36. & Karukot & 20.50 & 3 & MR & 11.75 & MS \\
\hline 37. & Karuvalli & 39.70 & 5 & MS & 13.75 & MS \\
\hline 38. & KaruthaNavara & 34.50 & 5 & MS & 12.75 & MS \\
\hline 39. & Kalyani & 20.70 & 3 & MR & 7.75 & MR \\
\hline 40. & Kullakar & 30.20 & 5 & MS & 13.25 & MS \\
\hline 41. & Kallondaikar & 43.10 & 5 & MS & 11.25 & MS \\
\hline 42. & Kattanoor & 34.90 & 5 & MS & 19.25 & $\mathrm{~S}$ \\
\hline 43. & Kalinga & 21.60 & 3 & MR & 14.50 & MS \\
\hline 44. & Koltara samba & 19.75 & 3 & MR & 13.50 & MS \\
\hline 45. & Kothamalli samba & 22.60 & 3 & MR & 9.80 & MR \\
\hline 46. & Kichali samba & 22.10 & 3 & MR & 16.25 & $\mathrm{~S}$ \\
\hline 47. & Kayamma & 40.20 & 5 & MS & 11.75 & MS \\
\hline 48. & Kuliyadichan & 30.90 & 5 & MS & 13.75 & MS \\
\hline 49. & Kerala kandasala & 20.80 & 3 & MR & 8.25 & MR \\
\hline 50. & Kalakeerai & 35.50 & 3 & MR & 9.25 & MR \\
\hline 51. & Maranellu & 60.50 & 7 & $\mathrm{~S}$ & 12.25 & MS \\
\hline 52. & Mallikar & 30.50 & 5 & MS & 11.75 & MS \\
\hline 53. & Mattai & 19.60 & 3 & MR & 11.75 & MS \\
\hline 54. & Molikarumbu & 22.50 & 3 & MR & 20.25 & $\mathrm{~S}$ \\
\hline 55. & Mapillai samba & 40.60 & 5 & MS & 9.75 & MR \\
\hline 56. & Mulampunchan & 20.50 & 3 & MR & 7.75 & MR \\
\hline 57. & Navarai & 35.60 & 5 & MS & 13.25 & MS \\
\hline 58. & Navarai Black & 30.80 & 5 & MS & 11.75 & MS \\
\hline 59. & Navarai Black 5571 & 22.50 & 3 & MR & 8.25 & MR \\
\hline 60. & Navarai Black 957 & 49.50 & 5 & MS & 14.75 & MS \\
\hline 61. & Navarai Black 6263 & 70.50 & 7 & $\mathrm{~S}$ & 19.75 & $\mathrm{~S}$ \\
\hline 62. & Noothipattu & 45.50 & 5 & MS & 14.00 & MS \\
\hline
\end{tabular}




\begin{tabular}{|c|c|c|c|c|c|c|}
\hline 63. & Norugan & 22.20 & 3 & MR & 9.25 & MR \\
\hline 64. & Ohenellu 6305 & 30.40 & 5 & MS & 9.25 & MR \\
\hline 65. & Oheruchittini & 30.60 & 5 & MS & 8.75 & MR \\
\hline 66. & Pattani & 43.50 & 5 & MS & 13.75 & MS \\
\hline 67. & Purple puttu & 9.10 & 1 & $\mathrm{R}$ & 4.96 & $\mathrm{R}$ \\
\hline 68. & Poongar & 20.10 & 3 & MR & 9.50 & MR \\
\hline 69. & Rajalakshmi & 69.50 & 7 & $\mathrm{~S}$ & 18.50 & $\mathrm{~S}$ \\
\hline 70. & Sadabahar & 30.50 & 5 & MS & 12.75 & MS \\
\hline 71. & Shabhagidan & 31.90 & 5 & MS & 8.50 & MR \\
\hline 72. & Seeraga samba & 24.00 & 3 & MR & 9.62 & MR \\
\hline 73. & Surakuruvai & 42.50 & 5 & MS & 13.10 & MS \\
\hline 74. & Swarna & 20.40 & 3 & MR & 9.50 & MR \\
\hline 75. & Swarnamalli & 32.30 & 5 & MS & 14.25 & MS \\
\hline 76. & Swarnamasuri & 21.60 & 3 & MR & 4.38 & $\mathrm{R}$ \\
\hline 77. & Thondi & 22.50 & 3 & MR & 9.75 & MR \\
\hline 78. & Thuyamalli & 21.50 & 3 & MR & 12.75 & MS \\
\hline 79. & Uma & 20.50 & 3 & MR & 9.50 & MR \\
\hline 80. & Varakuranellu & 35.10 & 5 & MS & 16.75 & $\mathrm{~S}$ \\
\hline 81. & Vanaprabha & 22.10 & 3 & MR & 13.75 & MS \\
\hline 82. & Vattan 5052 & 60.50 & 7 & $\mathrm{~S}$ & 16.25 & $\mathrm{~S}$ \\
\hline 83. & Veethiruppu & 9.50 & 1 & $\mathrm{R}$ & 9.75 & MR \\
\hline 84. & Virendra & 42.50 & 5 & MS & 9.25 & MR \\
\hline 85. & White ponni & 41.20 & 5 & MS & 11.75 & MS \\
\hline 86. & Thamarai & 18.00 & 3 & MR & 9.75 & MR \\
\hline 87. & Salem sannam & 30.80 & 5 & MS & 12.75 & MS \\
\hline 88. & Vasanai samba & 41.30 & 5 & MS & 13.20 & MS \\
\hline 89. & Anna 4 & 20.90 & 3 & MR & 7.51 & MR \\
\hline 90. & Chenellu 6805 & 73.90 & 7 & $\mathrm{~S}$ & 12.50 & MS \\
\hline 91. & Chungamnellu & 43.70 & 5 & MS & 14.50 & MS \\
\hline 92. & Kodikannan & 47.60 & 5 & MS & 16.25 & $\mathrm{~S}$ \\
\hline 93. & Varapudha & 19.60 & 3 & MR & 8.25 & MR \\
\hline 94. & Krishna hemarathi & 31.50 & 5 & MS & 16.95 & $\mathrm{~S}$ \\
\hline 95. & Jaya & 20.90 & 3 & MR & 13.25 & MS \\
\hline 96. & Abiyan & 40.70 & 5 & MS & 7.50 & MR \\
\hline 97. & Namchenbyeo & 24.50 & 3 & MR & 6.15 & MR \\
\hline 98. & Gowni & 21.50 & 3 & MR & 8.75 & MR \\
\hline 99. & Karsamba & 46.60 & 5 & MS & 12.22 & MS \\
\hline 100. & White sannam & 68.90 & 7 & $\mathrm{~S}$ & 19.25 & $\mathrm{~S}$ \\
\hline
\end{tabular}

PDI - Per cent Disease Index $\quad$ MR - Moderately Resistant S - Susceptible

I -Immune HS - Highly Susceptible LL - Lesion length

R - Resistant $\quad$ MS - Moderately Susceptible 
Table.4 Screening of rice germplasm for $X a 21, x a 13, x a 5$ and $X a 4$ genes using SSR markers

\begin{tabular}{|c|c|c|c|c|c|c|c|c|}
\hline \multirow[t]{2}{*}{ S.no } & \multirow[t]{2}{*}{ Germplasms } & \multicolumn{7}{|c|}{ Gene status } \\
\hline & & $\begin{array}{l}\text { RM21 } \\
(\text { Xa21 })\end{array}$ & $\begin{array}{l}\text { RM230 } \\
\text { (xa13) }\end{array}$ & $\begin{array}{l}\text { RM264 } \\
\text { (xa13) }\end{array}$ & $\begin{array}{l}\text { RM122 } \\
(x a 5)\end{array}$ & $\begin{array}{l}\text { RM164 } \\
(x a 5)\end{array}$ & $\begin{array}{l}\text { RM224 } \\
\text { (Xa4) }\end{array}$ & $\begin{array}{l}\text { RM167 } \\
\text { (Xa4) }\end{array}$ \\
\hline 1 & $\begin{array}{l}\text { TN } 1 \text { (Susceptible } \\
\text { check) }\end{array}$ & -- & -- & -- & -- & -- & -- & -- \\
\hline 2 & $\begin{array}{l}\text { IRBB60 (Resistant } \\
\text { check) }\end{array}$ & ++ & ++ & ++ & ++ & ++ & ++ & ++ \\
\hline 3 & Adukan & -- & -- & ++ & ++ & ++ & ++ & ++ \\
\hline 4 & Aman & -- & -- & ++ & -- & -- & ++ & ++ \\
\hline 5 & Anjali & -- & -- & ++ & -- & -- & ++ & -- \\
\hline 6 & Annada & -- & ++ & -- & -- & -- & ++ & -- \\
\hline 7 & Aryan 917 & -- & -- & -- & -- & -- & -- & -- \\
\hline 8 & Aryan 1023 & -- & -- & -- & -- & -- & ++ & -- \\
\hline 9 & Aryan 1102 & -- & ++ & -- & -- & -- & ++ & -- \\
\hline 10 & Aryan 1203 & ++ & -- & -- & ++ & -- & ++ & -- \\
\hline 11 & Aryan 5532 & -- & ++ & -- & ++ & -- & ++ & -- \\
\hline 12 & Aryan 6333 & -- & -- & -- & ++ & -- & -- & -- \\
\hline 13 & Bharathi & -- & -- & -- & ++ & ++ & -- & -- \\
\hline 14 & Bommi & ++ & ++ & -- & -- & -- & -- & -- \\
\hline 15 & Chemban 986 & -- & ++ & -- & -- & -- & -- & -- \\
\hline 16 & Chandikar & -- & ++ & -- & -- & -- & -- & -- \\
\hline 17 & Chembaru 5599 & -- & ++ & ++ & -- & -- & -- & -- \\
\hline 18 & Chembaru 4331 & -- & -- & -- & -- & -- & -- & -- \\
\hline 19 & ChittiraiKar & -- & ++ & -- & -- & -- & ++ & -- \\
\hline 20 & Chiruchittini 882 & -- & -- & -- & ++ & -- & ++ & -- \\
\hline 21 & Chenellu 4735 & -- & ++ & -- & ++ & ++ & ++ & -- \\
\hline 22 & Chenellu 5590 & -- & ++ & ++ & ++ & -- & ++ & -- \\
\hline 23 & Chenthodi & -- & -- & ++ & -- & -- & -- & -- \\
\hline 24 & $\begin{array}{l}\text { Chenkayamma } \\
5523\end{array}$ & -- & -- & ++ & -- & ++ & ++ & -- \\
\hline 25 & Chittini 1123 & -- & -- & ++ & -- & -- & -- & -- \\
\hline 26 & Chittini 5520 & -- & ++ & -- & -- & ++ & -- & -- \\
\hline 27 & $\begin{array}{l}\text { Chuvannachittini } \\
7135\end{array}$ & -- & -- & -- & -- & ++ & -- & ++ \\
\hline 28 & Chomala 826 & -- & -- & -- & -- & -- & -- & ++ \\
\hline 29 & $\begin{array}{l}\text { Company } \\
\text { thavalaikannan }\end{array}$ & -- & -- & -- & -- & -- & -- & -- \\
\hline 30 & Dhalakeera & ++ & ++ & -- & -- & -- & ++ & ++ \\
\hline 31 & Jai sree ram & -- & -- & -- & -- & ++ & -- & -- \\
\hline 32 & Kunjukunju 7168 & -- & -- & ++ & -- & -- & ++ & -- \\
\hline 33 & Kunjukunju 6974 & -- & -- & ++ & -- & -- & ++ & -- \\
\hline 34 & Kunjukunju 1811 & ++ & -- & ++ & -- & -- & ++ & -- \\
\hline
\end{tabular}




\begin{tabular}{|c|c|c|c|c|c|c|c|c|}
\hline 35 & Karnellu & -- & -- & ++ & -- & -- & -- & -- \\
\hline 36 & Karukot & -- & -- & ++ & -- & ++ & -- & -- \\
\hline 37 & Karuvalli & -- & -- & ++ & -- & -- & -- & ++ \\
\hline 38 & KaruthaNavara & -- & -- & ++ & -- & -- & -- & -- \\
\hline 39 & Kalyani & ++ & -- & ++ & ++ & ++ & ++ & -- \\
\hline 40 & Kullakar & -- & -- & ++ & ++ & -- & -- & -- \\
\hline 41 & Kallondaikar & ++ & -- & -- & ++ & -- & -- & -- \\
\hline 42 & Kattanoor & -- & -- & -- & ++ & ++ & -- & -- \\
\hline 43 & Kalinga & ++ & ++ & -- & ++ & -- & -- & -- \\
\hline 44 & Koltara samba & ++ & ++ & -- & -- & -- & ++ & ++ \\
\hline 45 & Kothamalli samba & ++ & -- & -- & ++ & -- & ++ & -- \\
\hline 46 & Kichali samba & -- & ++ & -- & -- & -- & ++ & -- \\
\hline 47 & Kayamma & -- & -- & -- & ++ & -- & -- & -- \\
\hline 48 & Kuliyadichan & -- & ++ & -- & -- & -- & -- & -- \\
\hline 49 & Kerala kandasala & -- & ++ & -- & -- & -- & ++ & -- \\
\hline 50 & Kalakeerai & ++ & ++ & -- & -- & -- & ++ & -- \\
\hline 51 & Maranellu & -- & -- & -- & -- & -- & -- & -- \\
\hline 52 & Mallikar & ++ & -- & -- & -- & -- & -- & -- \\
\hline 53 & Mattai & -- & -- & -- & -- & ++ & -- & -- \\
\hline 54 & Molikarumbu & -- & -- & -- & -- & -- & -- & -- \\
\hline 55 & Mapillai samba & -- & -- & ++ & -- & -- & ++ & -- \\
\hline 56 & Mulampunchan & -- & -- & -- & ++ & -- & -- & -- \\
\hline 57 & Navarai & -- & -- & -- & ++ & -- & ++ & -- \\
\hline 58 & Navarai Black & ++ & -- & -- & -- & -- & -- & -- \\
\hline 59 & $\begin{array}{l}\text { Navarai Black } \\
5571\end{array}$ & ++ & ++ & ++ & ++ & ++ & -- & ++ \\
\hline 60 & Navarai Black 957 & -- & -- & -- & -- & -- & ++ & -- \\
\hline 61 & $\begin{array}{l}\text { Navarai Black } \\
6263\end{array}$ & -- & -- & -- & -- & -- & -- & -- \\
\hline 62 & Noothipattu & -- & ++ & ++ & -- & -- & -- & -- \\
\hline 63 & Norugan & -- & ++ & ++ & -- & -- & -- & ++ \\
\hline 64 & Ohenellu 6305 & -- & ++ & -- & -- & -- & -- & -- \\
\hline 65 & Oheruchittini & -- & -- & -- & -- & -- & ++ & -- \\
\hline 66 & Pattani & -- & -- & -- & -- & ++ & -- & ++ \\
\hline 67 & Purple puttu & ++ & -- & ++ & -- & ++ & ++ & -- \\
\hline 68 & Poongar & ++ & -- & -- & ++ & -- & ++ & -- \\
\hline 69 & Rajalakshmi & -- & -- & -- & -- & -- & -- & -- \\
\hline 70 & Sadabahar & ++ & -- & -- & ++ & -- & -- & -- \\
\hline 71 & Shabhagidan & ++ & -- & -- & -- & -- & -- & -- \\
\hline 72 & Seeraga samba & ++ & -- & -- & -- & -- & ++ & -- \\
\hline 73 & Surakuruvai & ++ & -- & -- & -- & -- & -- & -- \\
\hline 74 & Swarna & ++ & -- & -- & -- & -- & ++ & -- \\
\hline 75 & Swarnamalli & -- & -- & -- & -- & -- & ++ & -- \\
\hline
\end{tabular}




\begin{tabular}{|l|l|l|l|l|l|l|l|l|}
\hline $\mathbf{7 6}$ & Swarnamasuri & ++ & ++ & ++ & -- & ++ & -- & -- \\
\hline $\mathbf{7 7}$ & Thondi & -- & ++ & ++ & -- & ++ & ++ & -- \\
\hline $\mathbf{7 8}$ & Thuyamalli & -- & ++ & -- & -- & -- & -- & -- \\
\hline $\mathbf{7 9}$ & Uma & ++ & ++ & ++ & -- & -- & ++ & -- \\
\hline $\mathbf{8 0}$ & Varakuranellu & -- & ++ & -- & -- & -- & -- & -- \\
\hline $\mathbf{8 1}$ & Vanaprabha & -- & ++ & ++ & -- & -- & ++ & -- \\
\hline $\mathbf{8 2}$ & Vattan 5052 & -- & -- & ++ & -- & -- & ++ & -- \\
\hline $\mathbf{8 3}$ & Veethiruppu & ++ & -- & ++ & -- & ++ & ++ & -- \\
\hline $\mathbf{8 4}$ & Virendra & ++ & -- & ++ & -- & ++ & -- & -- \\
\hline $\mathbf{8 5}$ & White ponni & ++ & -- & -- & -- & -- & ++ & -- \\
\hline $\mathbf{8 6}$ & Thamarai & -- & -- & -- & -- & -- & ++ & -- \\
\hline $\mathbf{8 7}$ & Salem sannam & ++ & ++ & -- & -- & -- & -- & -- \\
\hline $\mathbf{8 8}$ & Vasanai samba & ++ & ++ & ++ & -- & -- & -- & -- \\
\hline $\mathbf{8 9}$ & Anna 4 & -- & ++ & ++ & ++ & ++ & ++ & -- \\
\hline $\mathbf{9 0}$ & Chenellu 6805 & -- & -- & -- & -- & -- & ++ & -- \\
\hline $\mathbf{9 1}$ & Chungamnellu & -- & -- & -- & ++ & -- & -- & -- \\
\hline $\mathbf{9 2}$ & Kodikannan & -- & -- & ++ & ++ & -- & -- & -- \\
\hline $\mathbf{9 3}$ & Varapudha & -- & -- & ++ & -- & ++ & ++ & -- \\
\hline $\mathbf{9 4}$ & Krishna hemarathi & -- & ++ & -- & -- & -- & -- & -- \\
\hline $\mathbf{9 5}$ & Jaya & ++ & ++ & -- & -- & -- & -- & -- \\
\hline $\mathbf{9 6}$ & Abiyan & -- & ++ & -- & -- & -- & ++ & -- \\
\hline $\mathbf{9 7}$ & Namchenbyeo & -- & ++ & -- & -- & -- & ++ & -- \\
\hline $\mathbf{9 8}$ & Gowni & ++ & ++ & ++ & -- & -- & ++ & -- \\
\hline $\mathbf{9 9}$ & Karsamba & -- & ++ & -- & -- & -- & -- & -- \\
\hline $\mathbf{1 0 0}$ & White sannam & -- & -- & -- & -- & -- & -- & -- \\
\hline
\end{tabular}

Table.5 Multi-genic lines carrying different combinations of BLB resistant genes

\begin{tabular}{|c|c|c|l|}
\hline S.no & $\begin{array}{c}\text { Combination of BLB } \\
\text { resistant genes }\end{array}$ & $\begin{array}{c}\text { No.ofgermpl } \\
\text { asms }\end{array}$ & \multicolumn{1}{|c|}{ Name of the germplasms } \\
\hline $\mathbf{1}$ & $X a 21+x a 13+x a 5$ & 7 & $\begin{array}{l}\text { Kalyani, Kalinga, Navarai black 5571, Purple puttu, } \\
\text { Swarnamasuri, Veethiruppu, Virendra }\end{array}$ \\
\hline $\mathbf{2}$ & $X a 21+x a 13+X a 4$ & 10 & $\begin{array}{l}\text { Dhalakeera, Kunjukunju1811, Kalyani, Koltara } \\
\text { samba, Kalakeerai, Navarai black 5571, Purple puttu, } \\
\text { Uma, Veethiruppu, Gowni }\end{array}$ \\
\hline $\mathbf{3}$ & $X a 21+x a 5+X a 4$ & 7 & $\begin{array}{l}\text { Aryan 1203, Kalyani, Kothamalli samba, Navarai } \\
\text { black 5571, Purple puttu, Poongar, Veethiruppu }\end{array}$ \\
\hline $\mathbf{4}$ & $x a 13+x a 5+X a 4$ & 12 & $\begin{array}{l}\text { Adukan, Aryan 5532, Chenellu 4735, Chenellu 5590, } \\
\text { Chenkayamma 5523, Kalyani, Navarai black 5571, } \\
\text { Purple puttu, Thondi, Veethiruppu, Anna } \\
\text { Varapudha }\end{array}$ \\
\hline $\mathbf{5}$ & $X a 21+x a 13+x a 5+X a 4$ & 5 & $\begin{array}{l}\text { IRBB60, Kalyani, Navarai black 5571, Purple puttu, } \\
\text { Veethiruppu }\end{array}$ \\
\hline
\end{tabular}


Table.6 Details of the SSR primers used in present study, allele size (bp) and polymorphism information content (PIC)

\begin{tabular}{|c|c|c|c|c|}
\hline \multirow{2}{*}{ SSR Markers } & Total alleles & \multicolumn{2}{|c|}{ Allele size (bp) } & \multirow{2}{*}{ PIC } \\
\cline { 3 - 4 } & & Minimum & Maximum & \\
\hline RM21 & 6 & 100 & 210 & 0.688 \\
\hline RM230 & 5 & 240 & 280 & 0.507 \\
\hline RM264 & 5 & 150 & 200 & 0.664 \\
\hline RM164 & 6 & 240 & 300 & 0.501 \\
\hline RM224 & 5 & 240 & 280 & 0.392 \\
\hline RM167 & 6 & 100 & 160 & 0.603 \\
\hline Total & 5 & 100 & 150 & 0.345 \\
\hline Mean & 38 & 1170 & 1580 & 3.700 \\
\hline
\end{tabular}

Table.7 Composition of clusters formed in phylogenetic tree diagram for rice genotypes

\begin{tabular}{|c|c|c|}
\hline Cluster number & No.of genotypes & Name of the germplasms \\
\hline I & 25 & $\begin{array}{l}\text { Kattanoor, Bharathi, Mattai, Kullakar, Kayamma, } \\
\text { Kodikannan, Maranellu, Navarai black 6263, Poongar, } \\
\text { Aryan 1203, Sadabahar, Chungamnellu, } \\
\text { Mulampunchan, Navarai, Surakuruvai, Shabhagidan, } \\
\text { Rajalakshmi, Molikarumbu, Navarai black, White ponni, } \\
\text { Oheruchitteni, Chenellu 6805, Swarnamalli, TN 1, Uma }\end{array}$ \\
\hline II & 10 & $\begin{array}{l}\text { Pattani, Chuvannachitteni } 7135 \text {, Jai sree ram, Karuvalli, } \\
\text { Chomala 826, White sannam, Company thavalaikannan, } \\
\text { Chembaru 4331, Chemban 986, Aryan } 917\end{array}$ \\
\hline III & 25 & $\begin{array}{l}\text { Navarai black 5571, Adukan, Kalyani, Anna 4, Thondi, } \\
\text { Chenkayamma 5523, Varapudha, Veethiruppu, } \\
\text { Virendra, Swarnamasuri, Karukot, Chittini 5520, } \\
\text { Chenellu 4735, Vattan 5052, Kunjukunju 6974, Mapillai } \\
\text { samba, Karuthanavara, Karnellu, Kunjukunju 1811, } \\
\text { Aman, Anjali, Chittini 1123, Chenthodi, Ohenellu 6305, } \\
\text { IRBB } 60\end{array}$ \\
\hline IV & 40 & $\begin{array}{l}\text { Chittiraikar, Kunjukunju 7168, Annada, Chandikar, } \\
\text { Thamarai, Chiruchitteni 882, Aryan 6333, Norugan, } \\
\text { Aryan 1023, Abiyan, Kerala kandasala, Kichali samba, } \\
\text { Namchenbyeo, Chenellu 5590, Aryan 5532, } \\
\text { Varakuranellu, Kuliyadichan, Karsamba, Chembaru } \\
\text { 5599, Noothipathu, Krishna hemarathi, Thuyamalli, } \\
\text { Koltara samba, Dhalakeera, Kalakeerai, Bommi, Gowni, } \\
\text { Vanaprabha, Vasanai samba, Salem sannam, Kalinga, } \\
\text { Jaya, Purple puttu, Swarna, Seeraga samba, Navarai } \\
\text { black 957, Kothamalli samba, Mallikar, Kallondaikar, } \\
\text { Aryan 1102. }\end{array}$ \\
\hline
\end{tabular}




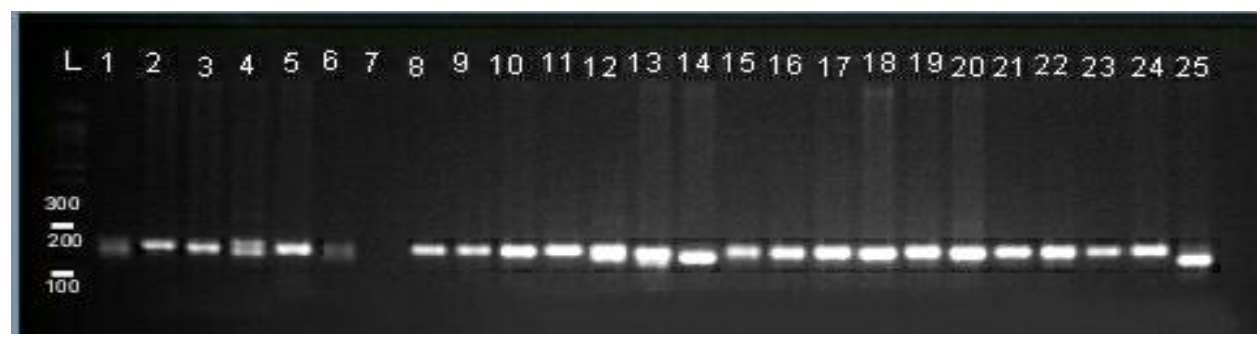

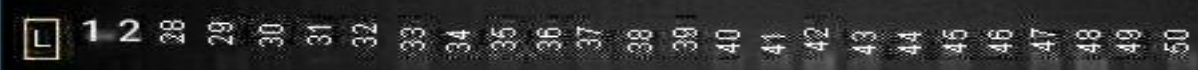

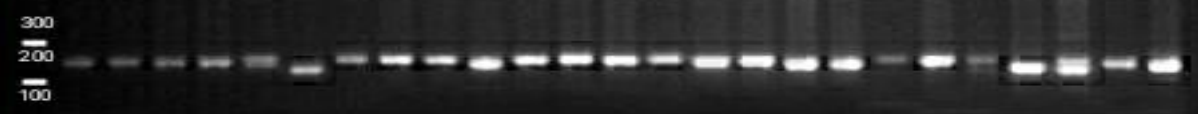

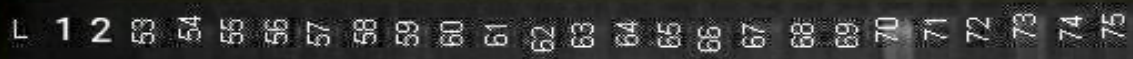

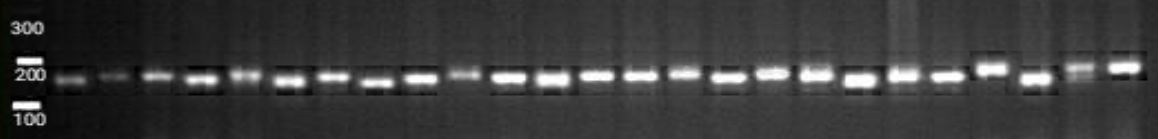

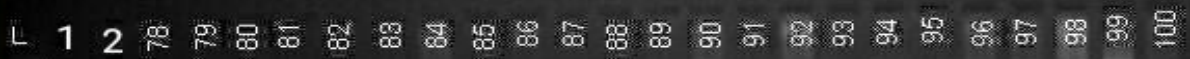

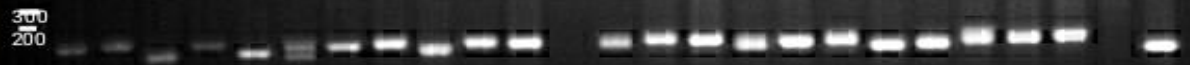
$\overline{100}$

Fig.1 Molecular profile of SSR marker RM224 -Xa4

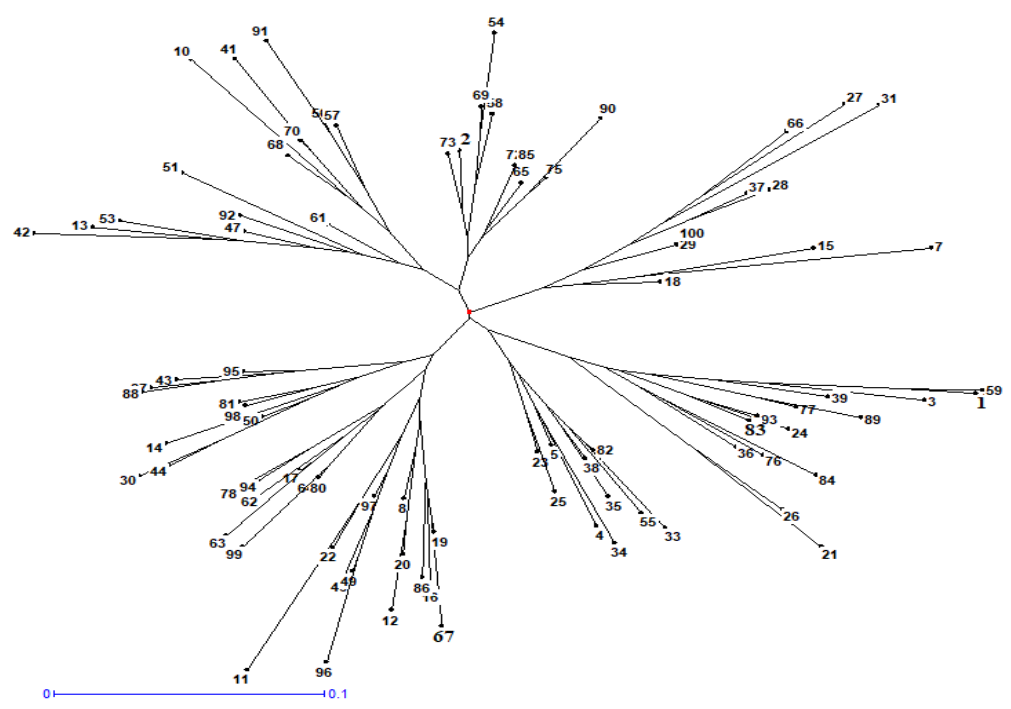

Fig.2 Phylogenetic tree constructed based on molecular marker (SSR) data 
The genotypes consists uprooted tree grouped 100 germplasm into four major clusters. Clusters I, II III and IV were consisting number of genotypes viz., 25, 10, 25 and 40. The UPMGA cluster tree analysis showed that the genotypes present in same cluster are genetically similar and different cluster are genetically dissimilar (Mubassir et al., 2016).

In the present study, bacterial leaf blight resistant genotypes were identified through polymorphic SSR markers. The BLB resistant genotypes were Veethiruppu, Purple puttu, Dhalakeera and Swarnamasuri. IRBB-60 (Positive check), Veethiruppu and Swarnamasuri genotypes were present in cluster III, Purple puttu and Dhalakeera were present in cluster IV. This showed genetic similarity and dissimilarity between the genotypes. The study result indicated that genetically diverse BLB resistant genotypes can be used for elite molecular breeding program and also used as a baseline for improvement of rice varieties.

\section{References}

Akhtar, M. A., Abdul Rafi, and Abdul Hameed. 2008. Comparison of methods of inoculation of Xanthomonas oryzae $p v$. oryzae in rice cultivars. Pakistan Journal of Botany 40 (5):2171-2175.

Arif, Muhammad, Muhammad Jaffar, Muhammad Babar, Munir A. Sheikh, Samina Kousar, Anjuman Arif, and Yusuf Zafar. 2008. Identification of bacterial blight resistance genes $\mathrm{Xa} 4$ in Pakistani rice germplasm using PCR. African Journal of Biotechnology 7 (5).

Banito, A., K. E. Kpémoua, and K. Wydra. 2010. "Screening of cassava genotypes for resistance to bacterial blight using strain $\times$ genotype interactions." Journal of Plant Pathology 92 (1):181-186.

Brondani, Claudio, Tereza Cristina Oliveira Borba, Paulo Hideo Nakano Rangel, and Rosana Pereira Vianello Brondani. 2006.
"Determination of genetic variability of traditional varieties of Brazilian rice using microsatellite markers." Genetics and Molecular Biology 29 (4):676-684.

Chen, Huilan, Shiping Wang, and Qifa Zhang. 2002. "New gene for bacterial blight resistance in rice located on chromosome 12 identified from Minghui 63, an elite restorer line." Phytopathology 92 (7):750-754.

Garcia, Antonio A. F., Luciana L. Benchimol, Antônia M. M. Barbosa, Isaias O. Geraldi, Cláudio L. Souza Jr, and Anete P. de Souza. 2004. "Comparison of RAPD, RFLP, AFLP and SSR markers for diversity studies in tropical maize inbred lines." Genetics and Molecular Biology 27 (4):579-588.

Gnanamanickam, S. S., V. Brindha Priyadarisini, N. N. Narayanan, Preeti Vasudevan, and S. Kavitha. 1999. "An overview of bacterial blight disease of rice and strategies for its management." Current Science 77 (11):1435-1444.

IRRI. 1996. Standard evaluation system for rice. The International Rice Research Institute, Manila, Philippines.

Jin, Liang, Yan Lu, Peng Xiao, Mei Sun, Harold Corke, and Jinsong Bao. 2010. "Genetic diversity and population structure of a diverse set of rice germplasm for association mapping." Theoretical and Applied Genetics 121 (3):475-487.

Kanawapee, Nantawan, Jirawat Sanitchon, Pranee Srihaban, and Piyada Theerakulpisut. 2011. "Genetic diversity analysis of rice cultivars (Oryza sativa L.) differing in salinity tolerance based on RAPD and SSR markers." Electronic Journal of Biotechnology 14 (6):2-2.

Kauffman, H. E. 1973. "An improved technique for evaluating resistance of rice varieties to Xanthomonas oryzae. Plant Disease Report 57:537-541.

Khush, G. S., and E. R. Angeles. 1999. A new gene for resistance to race 6 of bacterial blight in rice, Oryza sativa L. Rice Genetics Newsletter 16:92-93. 
Kihupi, A. N., E. R. Angeles, and G. S. Khush. 2001. Genetic analysis of resistance to bacterial blight, Xanthomonas oryzae pv. oryzae, in rice, Oryza sativa L. Euphytica 117 (1):39-46.

Kubo, Masayoshi, and Minjmaa Purevdorj. 2004. The future of rice production and consumption. Journal of Food Distribution Research 35 (856-201657064):128-142.

Kumar, Vikash, and Suresh Gopal Bhagwat. 2012. Microsatellite (SSR) based assessment of genetic diversity among the semi-dwarf mutants of elite rice variety WL112. International Journal of Plant Breeding and Genetics 6 (4):195-205.

Lang, Nguyen Thi, Bui Phuoc Tam, Nguyen Van Hieu, Chau Thanh Nha, Abdelbagi Ismail, Russell Reinke, and Bui Chi Buu. 2014. "Evaluation of rice landraces in Vietnam using SSR markers and morphological characters." SABRAO Journal of Breeding \& Genetics 46 (1).

Lee, K. S., S. Rasabandith, E. R. Angeles, and G. S. Khush. 2003. Inheritance of resistance to bacterial blight in 21 cultivars of rice. Phytopathology 93 (2):147-152.

Ma, Hui, Yu Yin, Zhi-Fu Guo, L. J. Cheng, Li Zhang, Ming Zhong, and Guo-Jun Shao. 2011. "Establishment of DNA fingerprinting of Liaojing series of japonica rice." Middle-East Journal of Scientific Research 8 (2):384-392.

Mubassir, M. H. M., Khondoker M. Nasiruddin, Nazmul Hoque Shahin, Shamsun Nahar Begum, Manas Kanti Saha, and A. Q. M. Bazlur Rashid. 2016. "SSR Marker Based Genetic Diversity Analysis of Some Rice Lines and Varieties for Bacterial Leaf Blight Resistance." Journal of Pharmaceutical Chemical and Biological Sciences 4 (4):475-486.

Nachimuthu, Vishnu Varthini, Raveendran Muthurajan, Sudhakar Duraialaguraja, Rajeswari Sivakami, Balaji Aravindhan Pandian, Govinthraj Ponniah, Karthika Gunasekaran, Manonmani Swaminathan, K. K. Suji, and Robin Sabariappan. 2015.
Analysis of population structure and genetic diversity in rice germplasm using SSR markers: an initiative towards association mapping of agronomic traits in Oryza sativa. Rice 8 (1):30.

Nagendran Krishnan, Gandhi Karthikeyan, Mohammed Faisal Peeran, Muthuraj Raveendran, Kuppusamy Prabakar, and Thiruvengadam Raguchander. 2013. "Management of bacterial leaf blight disease in rice with endophytic bacteria." World Applied Sciences Journal 28 (12):2229-2241.

Ni, Junjian, Peter M. Colowit, and David J. Mackill. 2002. "Evaluation of genetic diversity in rice subspecies using microsatellite markers." Crop science 42 (2):601-607.

Ogawa, T. 1996. "Monitoring race distribution and identification of genes for resistance to bacterial leaf blight." In Rice Genetics III: (In 2 Parts), 456-459. World Scientific.

Ogawa, T., Lao Lin, R. E. Tabien, and G. S. Khush. 1987. A new recessive gene for resistance to bacterial blight of rice. Rice Genetics Newsletter 4 (98): 100.

Perumalsamy, S., M. Bharani, M. Sudha, P. Nagarajan, L. Arul, R. Saraswathi, P. Balasubramanian, and J. Ramalingam. 2010. "Functional marker- assisted selection for bacterial leaf blight resistance genes in rice (Oryza sativa L.)." Plant Breeding 129 (4):400-406.

Petpisit, V., Gurdev S. Khush, and H. E. Kauffman. 1977. "Inheritance of Resistance to Bacterial Blight in Rice 1." Crop science 17 (4):551-554.

Rafi, Abdul, Abdul Hameed, Muhammad Afzal Akhtar, Syed Meher Ali Shah, Muhammad Junaid, Muhammad Shahid, and Syed Fahad Shah. 2013. "Field based assessment of rice bacterial leaf blight in major rice growing zones of Pakistan." Sarhad Journal of Agriculture 29 (3):415422.

Ramalingam, J., H. S. Basharat, and G. Zhang. 2001. "Polymorphism of DNA markers linked to bacterial blight resistance genes 
in useful rice germplasm." International Rice Research Notes (Philippines) 26 (2):23-24.

Ruan, Hui-Hui, Cheng-Qi Yan, De-Rong An, Ren-Hu Liu, and Jian-Ping Chen. 2008. "Identifying and Mapping New Gene $x a 32(\mathrm{t})$ for Resistance to Bacterial Blight (Xanthomonas oryzae pv. oryzae, Хoo) from Orza meyeriana L." Acta Agriculturae Boreali Occidentalis Sinica 17 (6):170-174.

Sajib, Abdul M., Md Hossain, Atmj Mosnaz, Hosneara Hossain, Md Islam, Md Ali, and Shamsul H. Prodhan. 2012. "SSR marker-based molecular characterization and genetic diversity analysis of aromatic landreces of rice (Oryza sativa L.). Journal of BioScience \& Biotechnology 1 (2).

Singh, K., Y. Vikal, S. Singh, H. Leung, H. S. Dhaliwal, and G. S. Khush. 2002. "40. Mapping of bacterial blight resistance gene xa8 using microsatellite markers." Rice Genetics Newsletter 19:94-97.

Singh, Nivedita, Debjani Roy Choudhury, Gunjan Tiwari, Amit Kumar Singh, Sundeep Kumar, Kalyani Srinivasan, R. K. Tyagi, A. D. Sharma, N. K. Singh, and Rakesh Singh. 2016. "Genetic diversity trend in Indian rice varieties: an analysis using SSR markers." BMC genetics 17 (1):127.

Sodhi, M., Yogesh Vikal, Maria Luz Caces George, G. S. Bala, G. S. Mangat, M. Garg, J. S. Sidhu, and H. S. Dhaliwal. 2003. "DNA fingerprinting and virulence analysis of Xanthomonas oryzae $p v$. oryzae isolates from Punjab, northern India." Euphytica 130 (1):107-115.

Song, Fengming, and Robert M. Goodman. 2001. "Molecular biology of disease resistance in rice." Physiological and Molecular Plant Pathology 59 (1):1-11.

Sonti, Ramesh V. 1998. "Bacterial leaf blight of rice: new insights from molecular genetics." Current Science 74 (3):206212.

Taura, Satoru, Tsugufumi Ogawa, Atsushi Yoshimura, and Ryoichi Ikeda. 1992. "ldentification of a Recessive Resistance Gene to Rice Bacterial Blight of Mutant Line XM6, Oryza sativa L." Japanese Journal of Breeding 42 (1):7-13.

Upadhyay, Priti, C. N. Neeraja, C. Kole, and Vikas Kumar Singh. 2012. "Population structure and genetic diversity in popular rice varieties of India as evidenced from SSR analysis." Biochemical Genetics 50 (9-10):770-783.

Zhang, DongLing, HongLiang Zhang, XingHua Wei, YongWen Qi, MeiXing Wang, JunLi Sun, Li Ding, ShengXiang Tang, Yong Sheng Cao, and XiangKun Wang. 2007. "Genetic structure and diversity of Oryza sativa L. in Guizhou, China." Chinese Science Bulletin 52 (3):343-351.

Zhou, Hai-fei, Zhong-wen Xie, and Song Ge. 2003. Microsatellite analysis of genetic diversity and population genetic structure of a wild rice (Oryza rufipogon Griff.) in China. Theoretical and Applied Genetics 107 (2):332-339.

\section{How to cite this article:}

Ashiba. R., K. Eraivan Arutkani Aiyanathan, R. Kannan and Arumugam Pillai. M. 2020. Genotypic Assessment of Bacterial Leaf Blight Resistance in Indigenous Rice (Oryza sativa L.) Germplasm. Int.J.Curr.Microbiol.App.Sci. 9(07): 210-227. doi: https://doi.org/10.20546/ijcmas.2020.907.024 